\title{
Anatomy of the Orbit
}

\author{
Stefan Lieber ${ }^{1}$ Juan C. Fernandez-Miranda ${ }^{1}$ \\ ${ }^{1}$ Department of Neurosurgery, Stanford University Medical Center, \\ Palo Alto, California, United States \\ J Neurol Surg B 2020;81:319-332.
}

Address for correspondence Juan C. Fernandez-Miranda, MD, Department of Neurosurgery, Stanford University Medical Center, 875 Blake Wilbur Drive, Palo Alto, CA 94304, United States (e-mail: drjfm@stanford.edu).

The orbit is a paired, transversely oval, and cone-shaped osseous cavity bounded and formed by the anterior and middle cranial base as well as the viscerocranium. Its main contents are the anterior part of the visual system, globe and optic nerve, and the associated neural, vascular, muscular, glandular, and ligamentous structures required for oculomotion, lacrimation, accommodation, and sensation.

A complex stream of afferent and efferent information passes through the orbit, which necessitates a direct communication with the anterior and middle cranial fossae, the pterygopalatine and infratemporal fossae, as well as the aerated adjacent frontal, sphenoidal, and maxillary sinuses and the nasal cavity.

This article provides a detailed illustration and description of the microsurgical anatomy of the orbit, with a focus on the intrinsically complex spatial relationships around the annular tendon and the superior orbital fissure, the transition from cavernous sinus to the orbital apex. Sparse reference will be made to surgical approaches, their indications or limitations, since they are addressed elsewhere in this special issue. Instead, an attempt has been made to highlight anatomical structures and elucidate concepts most relevant to safe and effective transcranial, transfacial, transorbital, or transnasal surgery of orbital, periorbital, and skull base pathologies.

\section{Osteology of the Orbit}

The orbit is a paired, transversely oval, and cone-shaped osseous cavity. When measured from the supraorbital foramen to the anterior genu of the cavernous internal carotid artery, its length in the anterior-posterior axis averages $63 \mathrm{~mm}{ }^{1}$

The orbital walls constitute a significant portion of the anterior and middle cranial base and are formed by seven bones: frontal, zygomatic, maxillary, lacrimal, ethmoid, palatine, and sphenoid (-Figs. 1-4). ${ }^{2}$

The orbital rim is formed by the frontal, zygomatic, and maxillary bones. In the superomedial aspect of the frontal bone, the supraorbital nerve and artery exit the orbit via the supraorbital notch or foramen. The zygomatic process of the frontal bone joins with the frontal process of the zygomatic bone at the frontozygomatic suture superolaterally. The maxillary bone forms most of the inferomedial rim of the orbit, and its frontal process meets the frontal bone at the frontomaxillary suture.

The orbital roof is composed of the orbital plate of the frontal bone anteriorly and the lesser wing of the sphenoid bone posteromedially. Two shallow depressions exist in the anterior orbit just behind the rim: superomedially, the trochlear fossa accommodates the trochlear apparatus, a ligamentous hypomochlion for the superior oblique muscle; superolaterally, the lacrimal gland rests in the lacrimal fossa. published online

September 17, 2020 (c) 2020 Georg Thieme Verlag KG Stuttgart · New York
DOI https://doi.org/ 10.1055/s-0040-1715096. ISSN 2193-6331. 


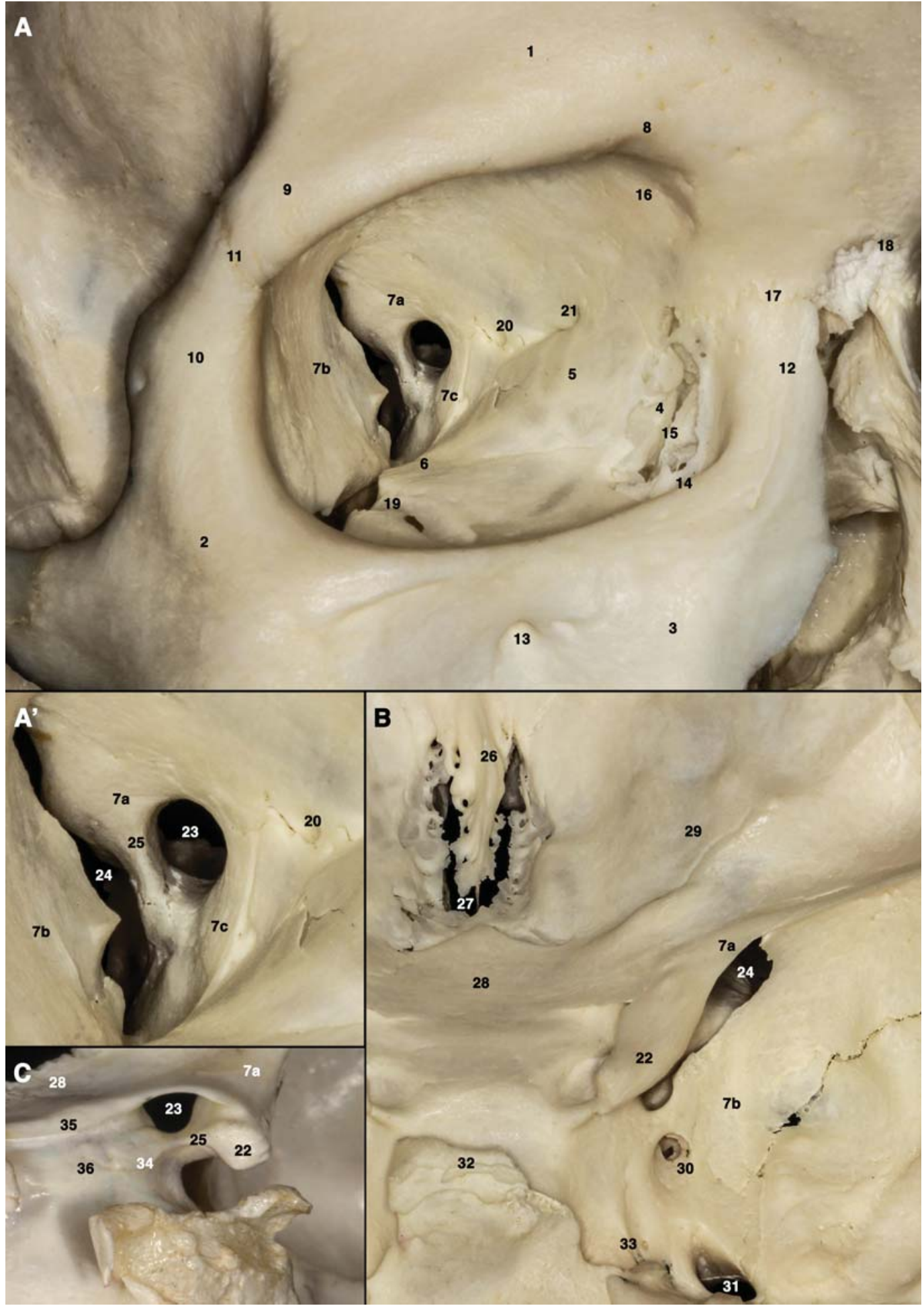

Fig. 1 Osteology of the Orbit I. (A) Oblique view of a right orbit, oriented along the axis of the optic canal. The orbit is formed by seven bones (the lacrimal bone is missing in this specimen). ( $A^{\prime}$ ) Enlarged view of (A), detailing the optic canal and the superior orbital fissure. (B) Osteology of the anterior and middle cranial fossae. (C) Osteology of the sellar region, oblique view oriented along the axis of a right optic canal. (A-C: macerated skull specimen). 1, frontal bone; 2 , zygomatic bone; 3 , maxillary bone; 4 , middle turbinate (the lacrimal bone is missing in this specimen); 5 , ethmoid bone; 6 , orbital process of the palatine bone; 7, sphenoid bone: 7a lesser wing, 7b greater wing, 7c body; 8, supraorbital notch (or foramen); 9 , zygomatic process of the frontal bone; 10 , frontal process of the zygomatic bone; 11 , frontozygomatic suture; 12 , frontal process of the maxillary bone; 13 , infraorbital foramen; 14, anterior lacrimal crest (maxillary bone); 15, site of the posterior lacrimal crest (the lacrimal bone is missing in this specimen); 16 , trochlear fossa; 17 , frontomaxillary suture; 18 , frontonasal suture (nasion); 19 , infraorbital groove; 20 , posterior ethmoidal foramen; 21 , anterior ethmoidal foramen; 22, anterior clinoid process; 23, optic canal; 24, superior orbital fissure; 25 , optic strut; 26 , crista galli; 27 , cribriform plate of the ethmoid bone; 28 , planum sphenoidale; 29 , orbital plate of the frontal bone; 30 , foramen rotundum; 31 , foramen ovale; 32 , dorsum sellae; 33 , lingual process of the sphenoid bone; 34 , lateral tubercular crest; 35 , limbus sphenoidale; 36 , tuberculum sellae. 

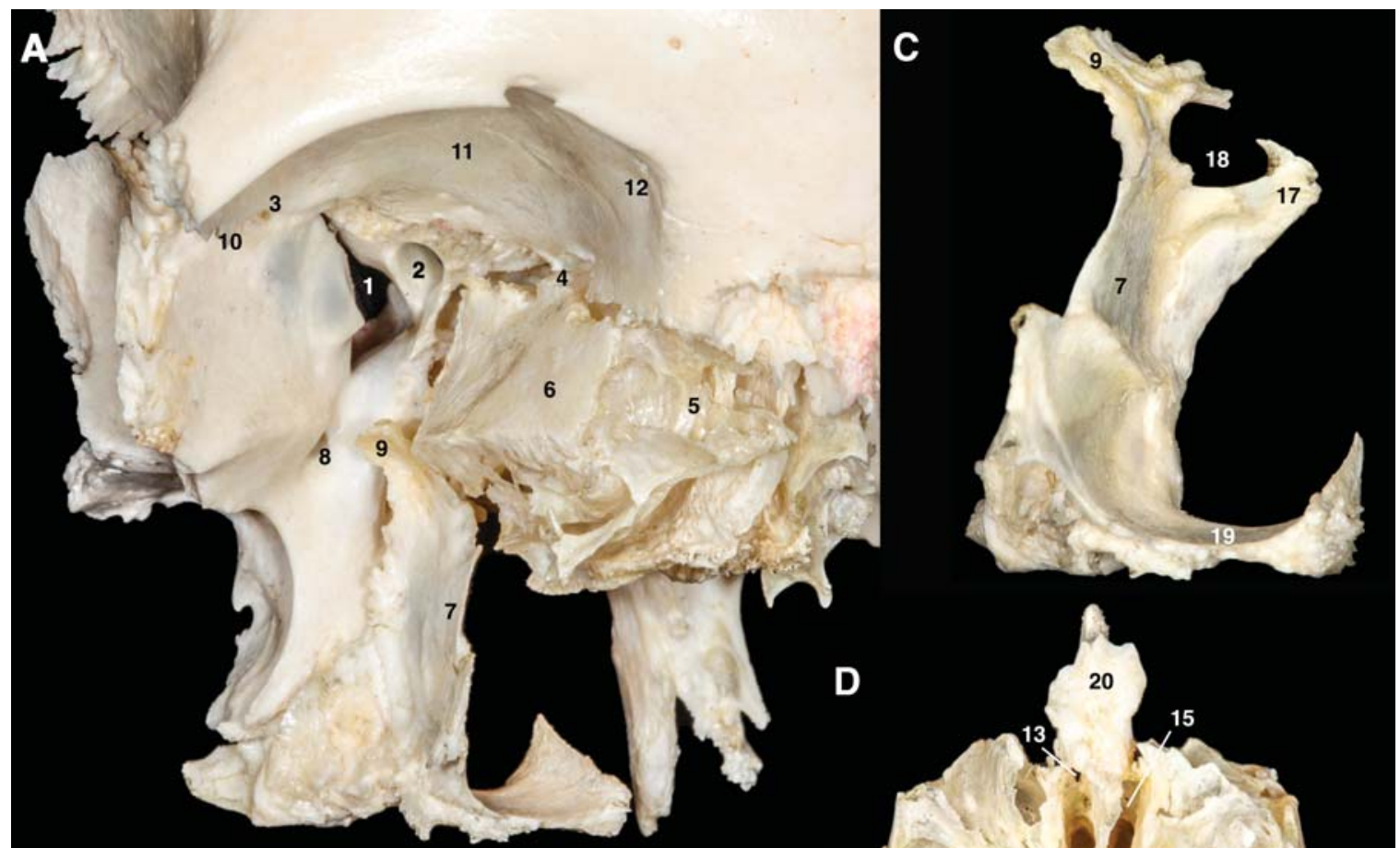

B

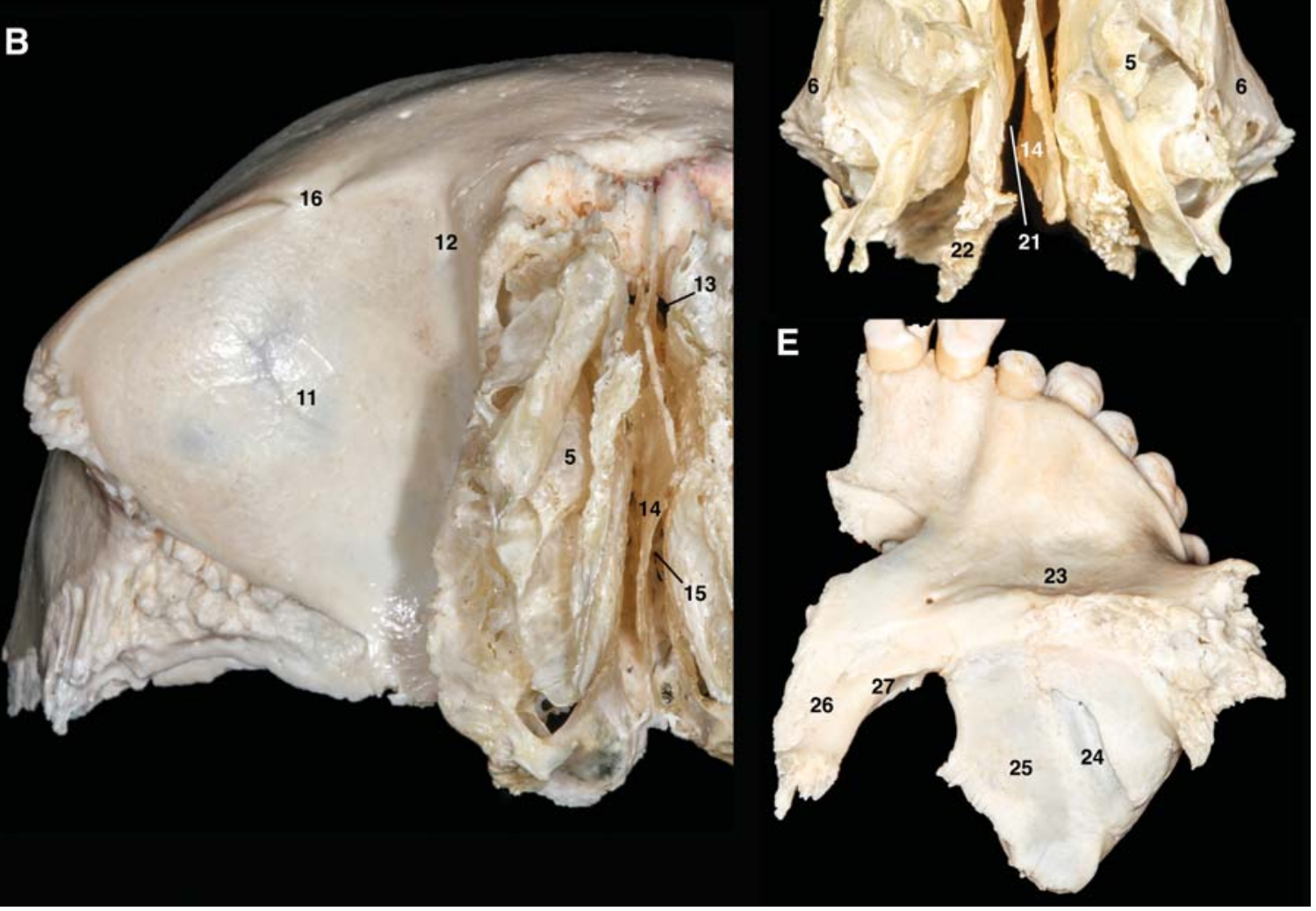

Fig. 2 Osteology of the Orbit II. (A) Antero-oblique view of a right orbit. The zygomatic, maxillary, and lacrimal bones have been removed for better visualization. (B) A right orbit as seen from below. (C) The palatine bone as seen from an anteromedial perspective, right side. (D) The ethmoid bone, anterior view. (E) The maxillary bone as seen from above, right side. (A-E: disarticulated macerated skull specimen). 1, superior orbital fissure; 2 , optic canal; 3 , lacrimal foramen; 4, frontoethmoidal suture; 5 , ethmoidal cells; 6 , orbital plate of the ethmoid bone (lamina papyracea); 7 , perpendicular plate of the palatine bone; 8 , foramen rotundum; 9 , orbital process of the palatine bone; 10 , frontosphenoidal suture; 11 , orbital plate of the frontal bone; 12 , trochlear fossa; 13 , foramen cecum; 14 , perpendicular plate of the ethmoid bone; 15 , cribriform plate of the ethmoid bone; 16 , supraorbital notch (or foramen); 17 , sphenoidal process of the palatine bone; 18 , sphenopalatine notch or foramen (leads to pterygopalatine fossa); 19 , horizontal plate of the palatine bone; 20 , crista galli; 21 , superior meatus; 22 , middle nasal concha; 23 , infraorbital foramen; 24 , infraorbital groove; 25 , orbital plate of the maxillary bone; 26 , frontal process of the maxillary bone; 27 , anterior lacrimal crest. 

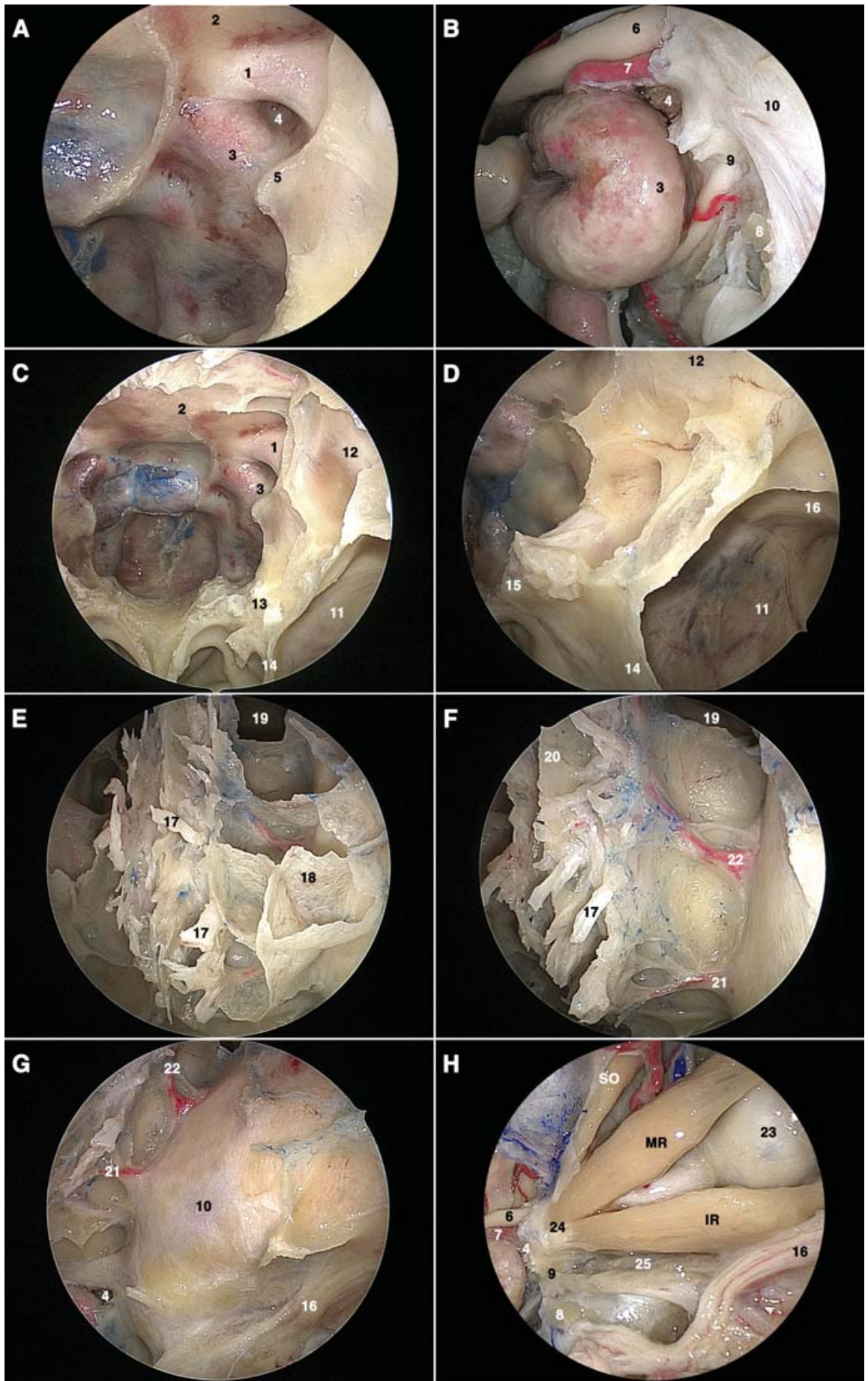

Fig. 3 Endoscopic endonasal perspective of the orbit. (A): Optic nerve, anterior ICA genu, and the lateral opticocarotid recess are important landmarks for reliable identification of the orbital apex, left side. (B): A, following complete bone removal. (C): Inferomedial aspect of the orbit, and spatial relationships with the maxillary sinus, the palatine bone, and the pterygopalatine fossa. (D): Enlarged view of (C). (E): Ethmoidal cells, olfactory fibers, and the cribriform plate, view toward the frontal base, centered to the left. (F): E after complete removal of the ethmoidal cells and the lamina papyracea to expose the ethmoidal arteries and the periorbita. (G): A left periorbita, 45 degrees optic. (H): $G$, following removal of periorbita and intraconal fat to expose the optic nerve, globe, and extraocular muscles. (A-H: formalin-fixed, silicone injected cadaveric specimen). ICA, internal carotid artery; IR, inferior rectus muscle; MR, medial rectus muscle; SO, superior oblique muscle; 1 , optic canal; 2 , planum sphenoidale; 3 , internal carotid artery (anterior genu); 4, lateral opticocarotid recess (which represents a pneumatized optic strut); 5 , sphenoid sinus (anterior wall); 6, optic nerve; 7, ophthalmic artery; 8, maxillary strut; 9, superior orbital fissure; 10, periorbita (continuation of periosteal dura mater); 11, maxillary sinus; 12 , orbital plate of the ethmoid bone (lamina papyracea); 13, sphenopalatine notch or foramen (leads to pterygopalatine fossa); 14 , perpendicular plate of the palatine bone (medial wall of the maxillary sinus); 15 , sphenoidal process of the palatine bone; 16 , infraorbital nerve; 17 , olfactory fibers (through the cribriform plate); 18 , ethmoidal cells; 19 , frontal sinus; 20 , perpendicular plate of the ethmoid bone; 21 , posterior ethmoidal artery and nerve; 22, anterior ethmoidal artery and nerve; 23, globe; 24 , annular tendon (or "annulus of Zinn"); 25, smooth orbital muscle (covering the inferior orbital fissure). 


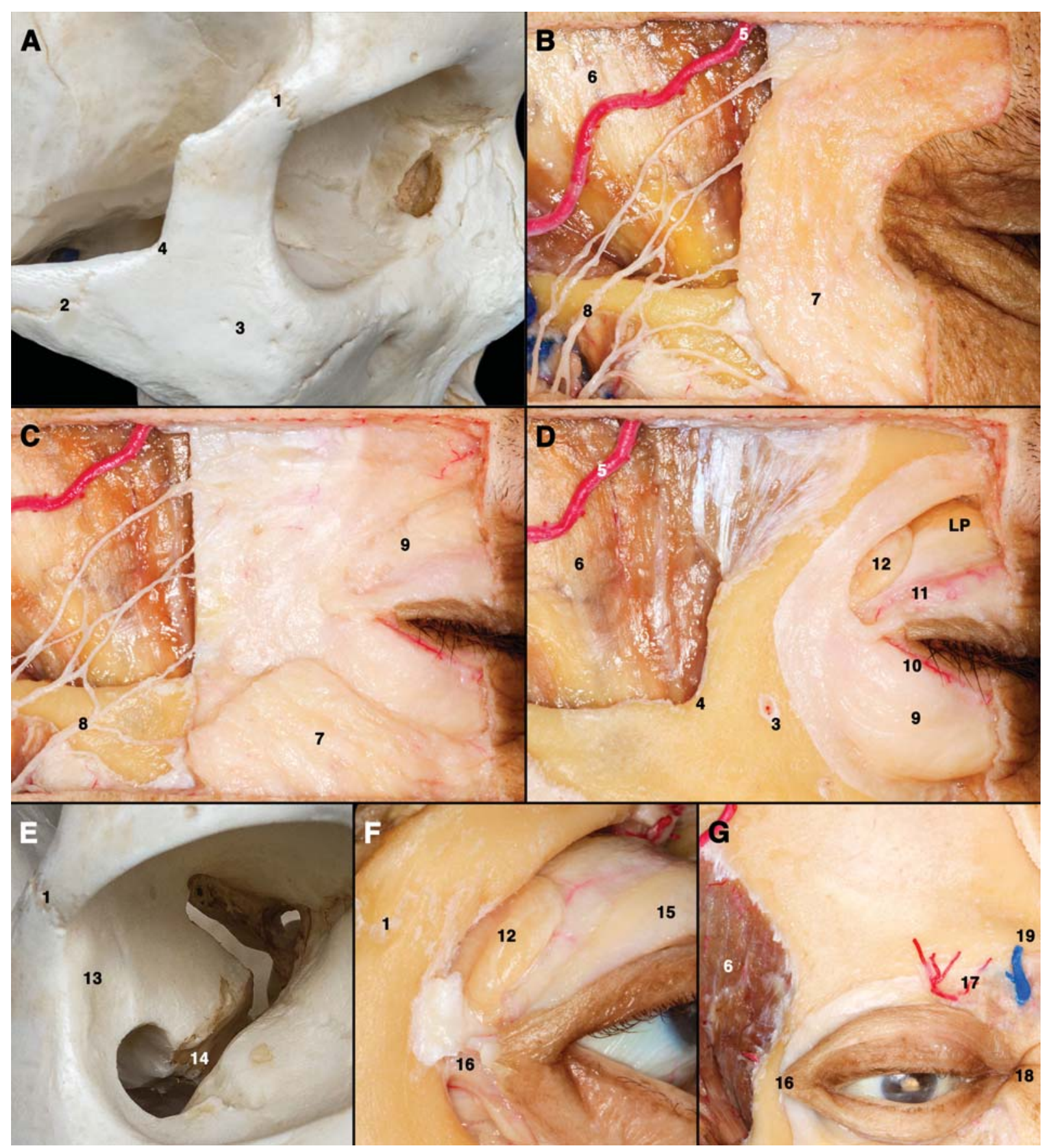

Fig. 4 Anterior orbital and periorbital space, orbital septum, lacrimal gland. (A) Osteology of the lateral orbit. (B-D) Stepwise illustration of facial nerve branches, orbicularis oculi muscle, orbital septum, the superior tarsal plate, and lacrimal gland. (E, F) Detail of the lateral orbital tubercle which serves as the attachment for the superior and inferior crura of the lateral canthal tendon. (G) Supraorbital artery and supratrochlear vein, exiting the orbit via the supraorbital notch and the trochlear fossa, respectively. (A, E: macerated skull specimen, B-D and F, G: formalin-fixed, silicone injected cadaveric specimen). 1, frontozygomatic suture; 2 , temporozygomatic suture; 3 , zygomaticofacial foramen or foramina; 4, jugal point (frontal process meets temporal process of the zygomatic bone); 5 , superficial temporal artery (frontal branch); 6 , temporalis muscle; 7, orbicularis oculi muscle (orbital part); 8, facial nerve (branches to the orbicularis oculi and frontalis muscles); 9, orbital septum; 10 , inferior lateral palpebral artery; 11 , superior lateral palpebral artery; 12 , lacrimal gland (orbital part); LP, levator palpebrae muscle; 13 , lateral orbital tubercle (or "tubercle of Whitnall"); 14 , inferior orbital fissure; 15 , superior tarsal plate (serves as the attachment for the aponeurosis for the levator palpebrae muscle); 16, superior and inferior crura of the lateral canthal tendon, lateral canthal ligament (lateral canthus); 17 , supraorbital artery; 18 , medial canthus; 19 , supratrochlear vein. 
The lateral wall is composed of the frontal process of the zygomatic bone anteriorly and the greater wing of the sphenoid posteriorly. The superior orbital fissure separates the lesser (superomedial) from the greater (inferolateral) wing of the sphenoid. Except for the lacrimal foramen (which transmits the recurrent meningeal artery, a branch of the ophthalmic artery) and the miniscule zygomatico-orbital foramina (which transmit the zygomaticofacial and zygomaticotemporal branches of the maxillary nerve), there are no osseous channels in the lateral wall.

The orbital floor is mostly formed by the orbital surface of the zygomatic bone anterolaterally and the orbital plate of the maxillary bone medially. The contribution of the orbital process of the palatine bone to the orbital floor is marginal; it is an important structure, however, which also partakes in the formation of the pterygopalatine fossa (anterior wall) and maxillary sinus (posterior wall). The inferior orbital fissure separates the orbital floor from the lateral wall, and the infraorbital groove emerges from it in a more medial trajectory toward the infraorbital canal, which transmits the infraorbital nerve and vessels.

The medial wall is far more complex: the lacrimal groove, which accommodates the lacrimal sac, is bounded by the anterior lacrimal crest of the maxillary bone and the posterior lacrimal crest of the lacrimal bone, and it opens into the nasal cavity inferomedially to transmit the nasolacrimal duct. More posteriorly, the lacrimal bone connects with the orbital plate of the ethmoid bone, better known as the lamina papyracea, and eventually the sphenoid bone. At the level of the frontoethmoidal suture, the anterior and posterior ethmoidal foramina (generally between one and four in number, but most commonly two) connect the orbit with the nasal cavity. ${ }^{3}$ They transmit their respective ethmoidal arteries (branches of the ophthalmic artery) and ethmoidal nerves (branches of the nasociliary nerve). When measured in the anterior-posterior axis, the anterior ethmoidal foramen is located $21 \mathrm{~mm}$ posterior to the anterior lacrimal crest of the maxillary bone, the posterior ethmoidal foramen $14 \mathrm{~mm}$ further posterior, and the optic canal another $6 \mathrm{~mm}$ posterior. ${ }^{3}$

The optic canal is formed by the body and lesser wing of the sphenoid bone. It is transversely oval-shaped, projects obliquely anterolaterally when viewed from its intracranial os, and opens into the superomedial aspect of the orbital apex. The roof of the optic canal is formed by an osseous bridge that extends from the limbus sphenoidale (body of the sphenoid) medially to the lesser wing laterally; the anterior clinoid process projects posterolaterally and forms the lateral wall of the optic canal, while the medial wall faces the sphenoid sinus.

The floor of the optic canal is formed by the optic strut, which continues to form the base of the anterior clinoid process and separates the optic canal from the superior orbital fissure. From the endonasal perspective, pneumatization of the base of the optic strut represents the lateral opticocarotid recess. The superior orbital fissure provides the communication between the middle cranial fossa and the orbit and is mostly bounded by the lesser wing of the sphenoid superomedially, the greater wing laterally, and the body of the sphenoid bone medially. The mid-portion of the lateral edge serves as a site of attachment for the annular tendon. The inferior aspect of the superior orbital fissure is separated from the foramen rotundum by the maxillary strut.

The inferior orbital fissure represents the incomplete border between the orbit's lateral wall, formed by the greater wing of the sphenoid bone, and the orbital floor, formed mostly by the orbital plate of the maxillary bone and, to a far lesser extent, the orbital process of the palatine bone. The inferior orbital fissure communicates with the pterygopalatine fossa posteromedially, and the infratemporal and temporal fossae anterolaterally.

\section{Anterior Orbital and Periorbital Space, Orbital Septum, and the Lacrimal Gland}

Underneath the skin, the orbicularis oculi muscle covers the circumference of the orbit; it consists of the orbital, preseptal, and pretarsal parts ( - Fig. 4) ${ }^{2,4}$ The orbital part arises from the osseous orbital rim, namely, the frontal, zygomatic, and maxillary bones and covers most of the orbit; it serves as the sphincter muscle of the eyelids and blends into the mimic musculature and the facial fascia peripherally. The eyelids accommodate the pretarsal part which is the continuation of the medial and lateral palpebral ligaments; its prime function is eye closure. The preseptal part lies between the orbital and pretarsal parts. The orbital septum underlies the orbicularis oculi muscle and represents the anterior border of the orbit. The superior and inferior tarsal plates shape the eye lids, and their medial and lateral extensions blend into the palpebral ligaments, the suspension of the eye lids. In the upper lid, the levator palpebrae muscle broadens into an aponeurosis which inserts broadly on the anterior surface of the superior tarsal plate. The medial canthal ligament divides to insert at the superior aspect of the anterior and posterior lacrimal crests, whereas the lateral canthal ligament inserts at the lateral orbital tubercle (or "tubercle of Whitnall"), an osseous prominence slightly posterior to the orbital rim and 10 to $12 \mathrm{~mm}$ posteroinferior to the frontozygomatic suture.

The lacrimal gland is an exocrine gland located in the lacrimal fossa, a depression in superolateral aspect of the orbit, considered a minor salivary gland. The gland consists of two lobes; the bigger orbital lobe lies superior and the smaller palpebral lobe deep to the aponeurosis of the levator palpebrae muscle.

The temporal and zygomatic branches of the facial nerve ascend over the mid-portion of the zygomatic arch to reach the frontalis and orbicularis oculi muscles. Sensation of the cornea, eyelids, and the periorbital region is transmitted by the nasociliary, supratrochlear, supraorbital, and lacrimal branches of the ophthalmic division of the trigeminal nerve, and the infraorbital, zygomaticofacial, and zygomaticotemporal branches of the maxillary nerve. ${ }^{1,5}$

\section{Orbital Apex, Annular Tendon, and the Extraocular Muscles}

The orbital apex is an immensely complex anatomical region: first, it represents the intersection of neurovascular 
structures entering from and exiting into the lateral compartment of the cavernous sinus and the optic canal with the orbital space (-Figs. 3, 5-7). ${ }^{1,2,5,6}$ Second, it is the site where the dura mater of the middle cranial fossa and the cavernous sinus blends into the dura mater of the superior orbital fissure, the optic nerve sheath, and the periorbita and forms an annular tendon from which the four rectus muscles arise. The annular tendon, also known as the "annulus of Zinn," encloses the optic nerve, the ophthalmic artery, and most cranial nerves associated with vision, oculomotion, and accommodation. The notable exceptions to this are the trochlear nerve, and the lacrimal and frontal nerves (branches of the ophthalmic division of the trigeminal nerve). The annular tendon is attached around the superior, inferior, and medial aspects of the optic canal and the most medial aspect of the lateral margin of the superior orbital fissure, formed by the sphenoid's greater wing. Since it only encircles the optic canal and the mid-portion of the superior orbital fissure, it in turn subdivides the superior orbital fissure into three separate compartments: a superolateral, a central, and an inferomedial compartment (-Fig. 6).

The superolateral compartment is traversed by the lacrimal and frontal nerves of the trigeminal nerve's ophthalmic branch, the trochlear nerve and the superior ophthalmic vein. The central compartment (or oculomotor foramen) contains all neurovascular structures that enter and exit the orbit through the annular tendon and course within the intraconal space. These are the superior and inferior divisions of the oculomotor nerve, the nasociliary nerve, the abducens nerve, and the parasympathetic and sensory fibers of the ciliary ganglion via the superior orbital fissure, as well as the optic nerve and the ophthalmic artery via the optic canal. The inferomedial compartment only accommodates the inferior ophthalmic vein and a varying number of branches of the carotid sympathetic plexus, the sympathetic contribution to the ciliary ganglion. The inferior boundary of the inferior compartment is formed by the remnant of the inferior smooth orbital muscle, which covers the entire length of the inferior orbital fissure, and thereby separates the orbit from the temporal, infratemporal, and pterygopalatine fossae. ${ }^{7}$ This muscle blends into the periosteum of the orbital floor. The infraorbital nerve, artery, and vein course underneath the orbital periosteum.

The superior, lateral, medial, and inferior rectus muscles arise from the annular tendon to form a muscular cone (-Figs. 5, 7 and 9). The annular tendon represents the proximal end, whereas the globe represents the anterior or distal end. This cone is sheathed by the periorbita, a thin membrane, and densely filled with fat invested with a delicate system of ligaments (Koornneef's bands) which surrounds the intraconal neurovascular contents.

The superior rectus muscle courses anteriorly to attach to the sclera of the globe, $\sim 7.5 \mathrm{~mm}$ posterior to the margin of the cornea. It courses directly underneath the levator palpebrae muscle; both muscles share a soft fascia.

The lateral rectus muscle originates from the annular tendon over the greater sphenoid wing, whereas the medial rectus muscle originates from the annular tendon lateral of the optic canal; they attach to the medial and lateral sclera, $\sim 7$ and $5.5 \mathrm{~mm}$ posterior to the corneal limbus, respectively.

The inferior rectus muscle courses anteriorly and pierces the retractor band of the lower lid (the capsulopalpebral fascia).

The superior oblique muscle does not originate from the annular tendon but from the periorbita superomedial to the optic canal. It courses anteriorly within the superomedial aspect of the intraconal space to reach the trochlear fossa. Here, its tendon is redirected by a ligamentous trochlea (trochlear hypomochlion), and it travels in a posteromedial direction underneath the superior rectus muscle to eventually insert on the sclera deep to the superior rectus belly.

The inferior oblique muscle is unique in that it does not originate from the annular tendon or orbital apex but from the inner aspect of the inferior orbital rim between the lacrimal fossa and the zygomaticomaxillary fissure. It courses laterally, inferior to the inferior rectus muscle to attach to the sclera deep to the lateral rectus muscle, at the area corresponding to the macula. $2,4,5$

\section{Cranial Nerves Associated with the Orbit and the Ciliary Ganglion}

\section{Special Sensory Innervation}

The optic nerve can be subdivided into five segments: intraocular, intraorbital, intracanalicular, preforaminal, and cisternal; the two last segments represent the intracranial segments. The optic nerve emerges from the optic chiasm and courses anteroinferolaterally, usually at a 30- to 45degree angle, within the chiasmatic cistern. In this segment, the optic nerve is transversely oval shaped, and its diameters average 5 by $3 \mathrm{~mm} .{ }^{1}$ The preforaminal segment refers to the short segment where the nerve runs underneath the falciform ligament, a dural fold extending from the limbus sphenoidale to the anterior clinoid process but has yet to enter the osseous optic canal (-Fig. 5). It is a short segment, measuring a mere 3 to $5 \mathrm{~mm}$, and is surrounded by cerebrospinal fluid. ${ }^{1}$ The optic nerve's intracanalicular segment passes through the proper osseous optic canal, whereas the intraorbital segment is densely surrounded by fat and the complex neurovasculature of the intraconal space. The intraocular segment represents the optic nerve within the sclera of the globe, also known as the optic disc.

Once the optic nerve emerges from its osseous canal, it retains its dura and arachnoid protective cover; therefore, a continuous subarachnoid space exists, extending from the most proximal cisternal segment to the sclera of the globe. As discussed earlier, the optic nerve's dural sheath blends into the periorbita and participates on the formation of the superomedial aspect of the annular tendon. ${ }^{2}$

\section{Motor Innervation}

The oculomotor nerve courses within its interdural cistern to enter the roof of the cavernous sinus just below the anterior clinoid process; it splits into superior and inferior divisions right before entering the orbit. Both divisions take a medial course just lateral to the optic strut to pass through 


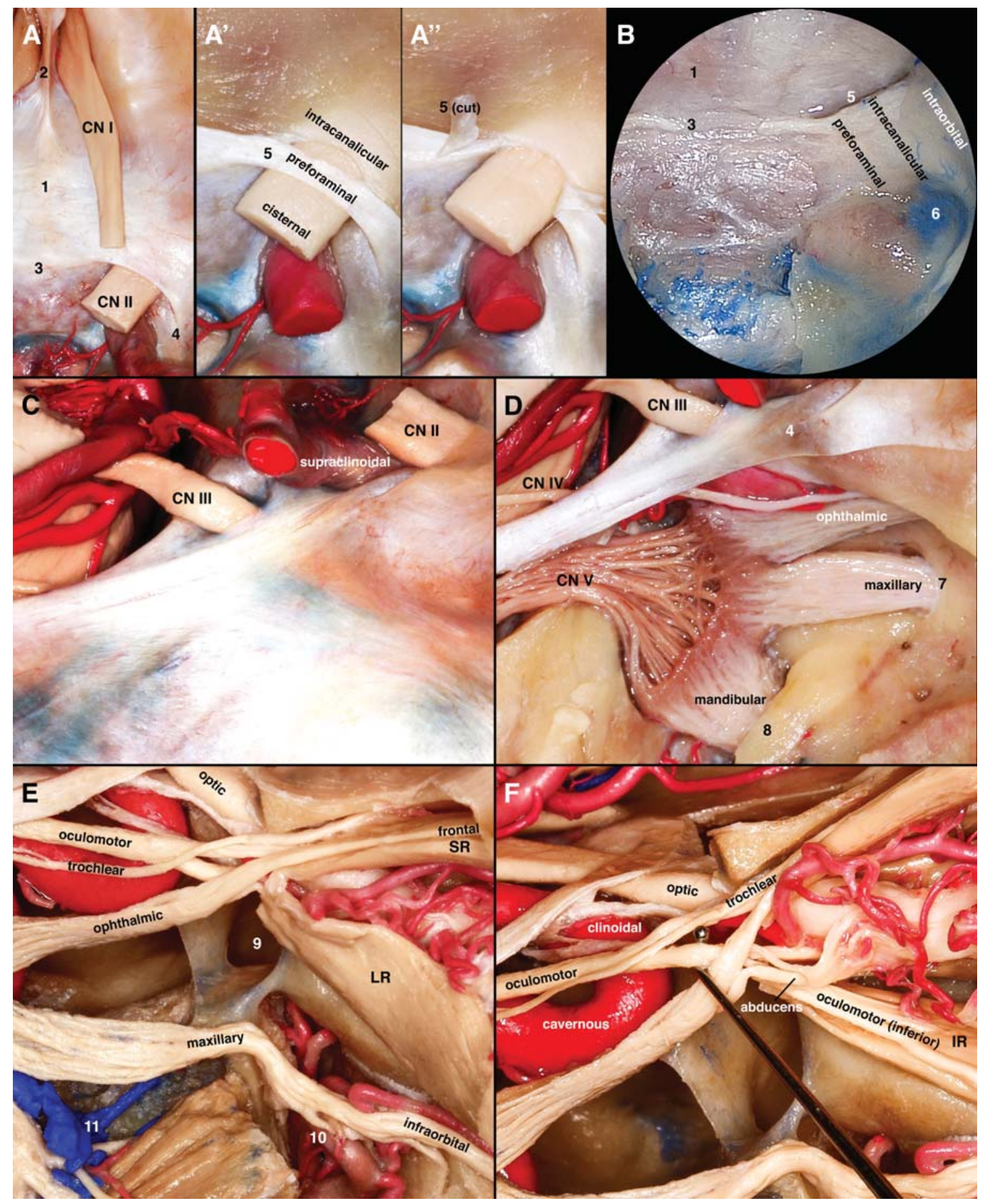

Fig. 5 Optic canal, falciform ligament, and the orbital apex. (A-A") Detail of the cisternal, preforaminal, intracanalicular, and intraorbital segments of the optic nerve from the transcranial and (B) endonasal perspective. (C-F) Stepwise illustration of the cavernous sinus, the superior orbital fissure, and the orbital apex. Lateral view, right side. (A-F: formalin-fixed, silicone injected cadaveric specimen). CN I, olfactory nerve; CN II, optic nerve; IR, inferior rectus muscle; LR, lateral rectus muscle; SR, superior rectus muscle; 1 , planum sphenoidale; 2 , falx cerebri; 3 , limbus sphenoidale; 4, anterior clinoid process; 5 , falciform ligament; 6 , lateral opticocarotid recess (the equivalent of the optic strut from the endonasal perspective); 7 , foramen rotundum; 8 , foramen ovale; 9 , sphenoid sinus; 10 , pterygopalatine fossa and branches of the internal maxillary artery; 11 , pterygoid venous plexus.

the central compartment of the superior orbital fissure (-Figs. 5-9).

The smaller superior division consists of the motor branches to the superior rectus and levator palpebrae muscles; these branches ascend lateral to the optic nerve and insert to the inner surface of their respective muscles, the latter after passing through the substance of the superior rectus. 


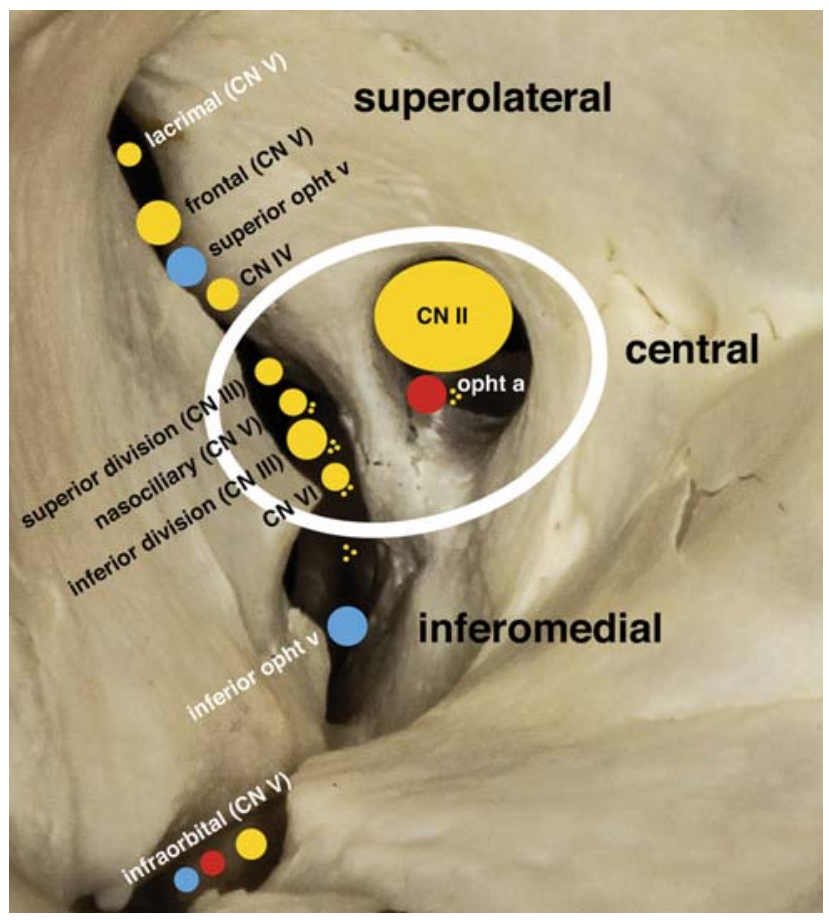

Fig. 6 The compartments of the superior orbital fissure. This schematic drawing is based on - Fig. 1A. The annular tendon (white ring) is attached around the superior, inferior, and medial aspects of the optic canal and the most medial aspect of the lateral margin of the superior orbital fissure. Since it only encircles the optic canal and the mid-portion of the superior orbital fissure, it in turn subdivides the superior orbital fissure into three separate compartments: a superolateral, a central, and an inferomedial.

The inferior division has a greater diameter and gives rise to motor branches to the medial rectus, inferior rectus and inferior oblique muscles, as well as to pupillary fibers (see paragraph on "ciliary ganglion").

The trochlear nerve is the only cranial nerve associated with oculomotion which does not enter the orbit through the annular tendon. It courses adjacent to and superomedial to the frontal nerve to eventually blend into the superior aspect of the superior oblique muscle.

\section{Sensory Innervation}

The first two branches of the trigeminal nerve are associated with the orbit. The ophthalmic nerve, the smallest branch of the trigeminal nerve, is embedded in the dura mater of the cavernous sinus' lateral wall and separates into the lacrimal, frontal, and nasociliary nerves before entering the superior orbital fissure.

The lacrimal nerve enters the orbit via the superolateral compartment of the superior orbital fissure and courses along the superior margin of the lateral rectus muscle toward the lacrimal gland. During its course, it receives postganglionic parasympathetic fibers ascending from the pterygopalatine ganglion via the zygomatic nerve.

The frontal nerve, a branch of the ophthalmic division of the trigeminal nerve, rests on the levator palpebrae muscle, immediately underneath the periorbita, having entered the orbit via the superolateral compartment; it divides into a supraorbital nerve and a supratrochlear nerve. The supraorbital nerve travels anteriorly along the medial aspect of the levator palpebrae and superior rectus muscles, accompanied by its respective arterial branch, to exit the orbit through a notch or foramen in the supraorbital rim. The smaller supratrochlear nerve courses more medially toward the trochlear fossa, again accompanied by its respective arterial branch, to pierce through the orbital septum to exit the orbit near the trochlea.

The nasociliary nerve is the only trigeminal branch to pass through the annular tendon and the central compartment of the superior orbital fissure. It gives rise to the sensory root of the ciliary ganglion (preganglionic parasympathetic fibers, see paragraph on "ciliary ganglion") and the long ciliary nerves, which transmit sympathetic fibers to the globe (-Figs. 6, 7 and 9). Both short and long ciliary nerves enter the sclera in proximity to the optic nerve, joined by the posterior ciliary arteries. The nasociliary nerve ascends over the optic nerve, coursing medially to give raise to the anterior and posterior ethmoidal nerves, which accompany their respective ethmoidal arteries to exit the orbit via the ethmoidal foramina, as well as to the infratrochlear nerve.

The ciliary ganglion is situated on the inferolateral aspect of the optic nerve, between the nerve and lateral rectus muscles, just anterior to the steep ascending turn of the ophthalmic artery(-Figs. 5, 7, and 9). As described in the previous sections, it receives a motor root, representing parasympathetic fibers running within the oculomotor nerve's inferior division to the inferior oblique muscle, and a sensory root, formed by sensory fibers from the nasociliary nerve (corneal sensation) and postganglionic sympathetic fibers (nerves to the pupillary dilator muscle). The latter ascend from the superior cervical ganglion, course within the carotid sympathetic plexus and reach the orbit via the ophthalmic artery and the ophthalmic and abducens nerves. Only the parasympathetic fibers synapse in the ciliary ganglion to be emitted as the short ciliary nerves responsible for pupillary constriction and accommodation, sympathetic fibers course within the long ciliary nerves. Both short and long ciliary nerves enter the sclera in proximity to the optic nerve, joined by the posterior ciliary arteries.

The maxillary nerve passes through foramen rotundum to enter the pterygopalatine fossa. It gives rise to preganglionic fibers to the sphenopalatine ganglion and the infraorbital and zygomatic nerves, both of which initially course anteriorly in the orbital floor, deep to the periorbita. The infraorbital nerve continues in the infraorbital groove and canal to exit via the infraorbital foramen of the maxillary bone, whereas the zygomatic nerve takes a more lateral course toward the orbital wall. Its terminal branches, the zygomaticofacial and zygomaticotemporal nerves exit via the zygomaticofacial foramina of the zygomatic bone (-Fig. 4).

The abducens nerve is unique in that it travels freely within the cavernous sinus and is not embedded in the dura mater of its lateral wall. It lies just medial to the ophthalmic nerve and enters the orbit through the annular tendon. It then turns laterally to insert to the inner surface of the lateral rectus muscle. ${ }^{2,5}$ 


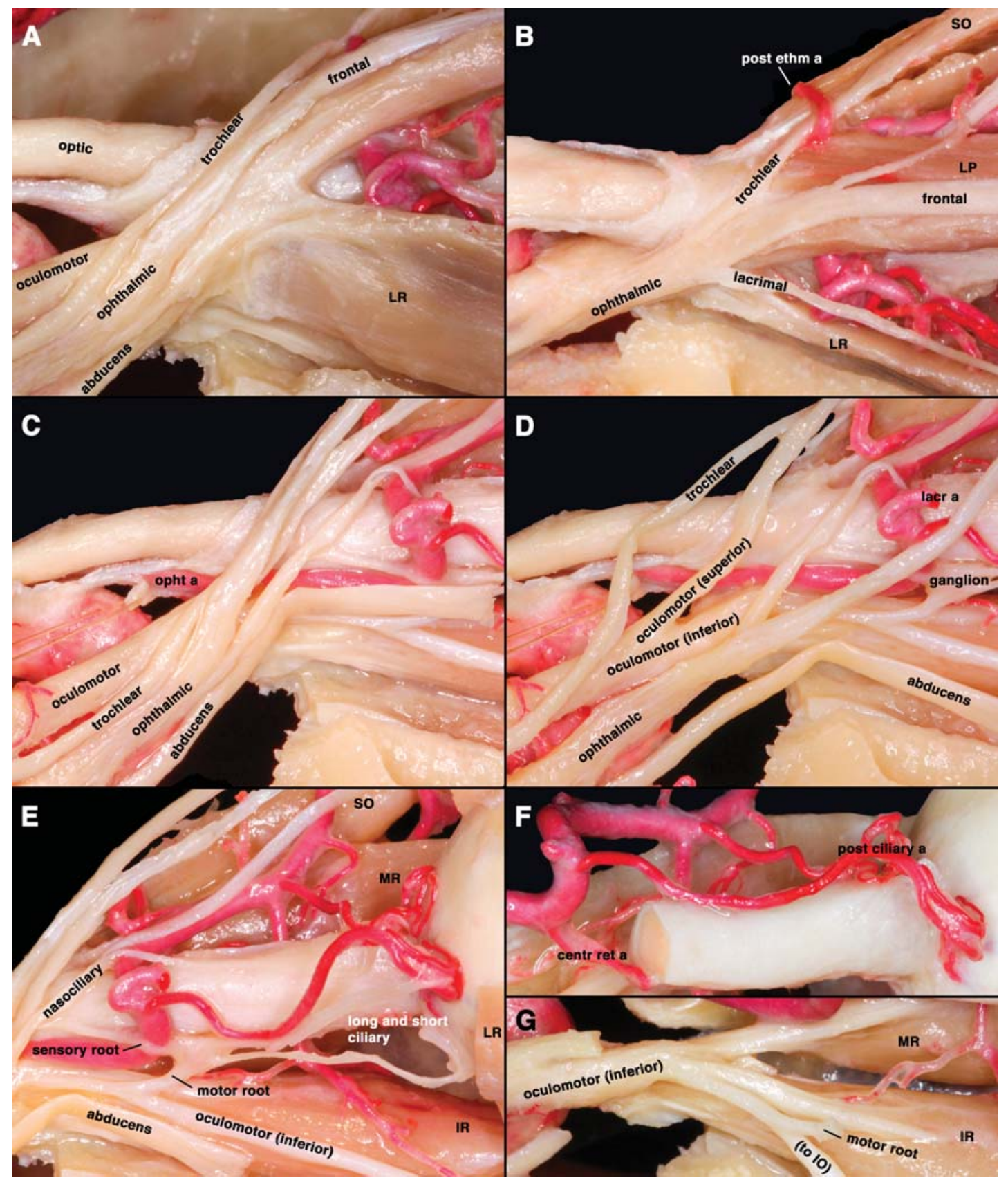

Fig. 7 Orbital apex and intraconal structures. This figure illustrates the complex microanatomy of neurovascular, ligamentous, dural, and muscular structures around the orbital apex. Bone, periorbita, and intraconal fat have largely been removed for visualization. The right side of a formalin-fixed, silicone injected cadaveric specimen is shown. (A) Superolateral view. (B) Superior view. (C) Superolateral view, the lateral rectus, superior rectus, and levator palpebrae muscles have been removed. (D) Similar to (C), but the nerves have been fanned out for visualization. (E) Lateral view, detail of the ciliary ganglion. (F) Lateral view, detail of the posterior ciliary arteries. (G) Lateral view, detail of the inferior division of the oculomotor nerve. centr ret a, central retinal artery; ganglion, ciliary ganglion; IO, inferior oblique muscle; IR, inferior rectus muscle; lacr a, lacrimal artery; long and short ciliary, long and short ciliary nerves (sympathetic and parasympathetic postganglionic fibers); LP, levator palpebraemuscle; LR, lateral rectus muscle; motor root, motor root branching off the inferior division of the oculomotor nerve; MR, medial rectus muscle; opht a, ophthalmic artery; post ciliary a, posterior ciliary arteries; post ethm a, posterior ethmoidal artery; sensory root, sensory root branching off the nasociliary nerve; SO, superior oblique muscle.

\section{Arterial Vasculature Associated with the Orbit}

The anterior genu of the internal carotid artery (ICA) consists of the cavernous ICA segment before the proximal dural ring, and the short, extracavernous clinoidal ICA segment between the proximal and distal dural rings., ${ }^{2,6}$ The clinoidal ICA is fixed to the anteromedial base of the optic strut by the proximal and distal dural rings, and the supraclinoidal ICA segment commences once the artery has passed through the distal dural ring to enter the intradural space (-Figs. $\mathbf{3}$ and $\mathbf{5}$ ). 


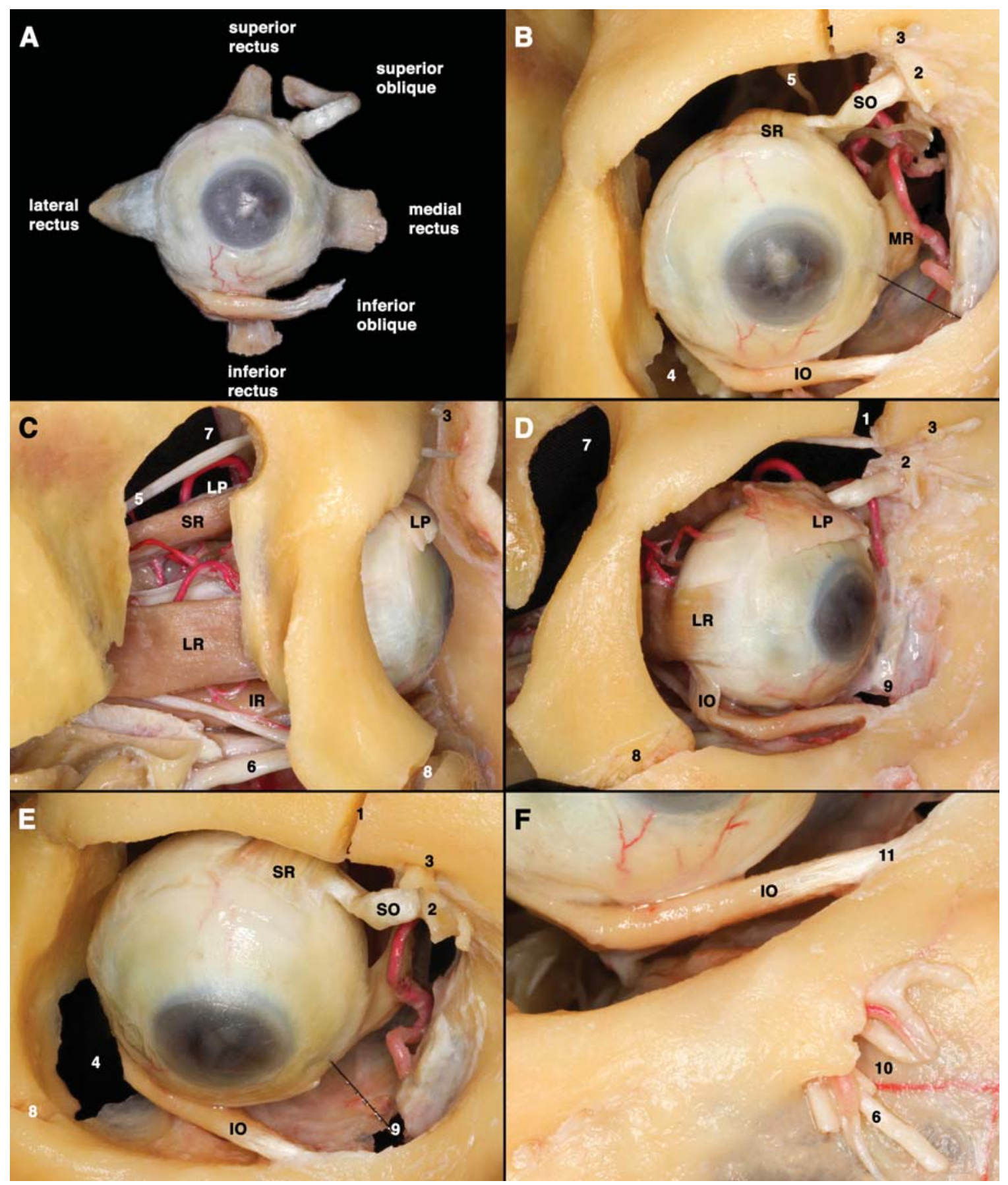

Fig. 8 Extraocular muscles. This figure illustrates the extraocular muscles and their respective sites of origin and attachment at the globe. Periorbita, intraconal fat, orbital septum, and preseptal structures have largely been removed for visualization. The osseous exposure corresponds to a one-piece frontotemporal-orbitozygomatic craniotomy which is initiated with a MacCarty burr hole. The right side of a formalinfixed, silicone injected cadaveric specimen is shown. (A), globe, as seen from anterior. (B) Anterior view, (C) lateral view, (D) oblique anterior view, (E) oblique superior view, (F) detail of the origin of the inferior oblique muscle at the anterior lacrimal crest. $I O$, inferior oblique muscle; IR, inferior rectus muscle; LP, levator palpebrae muscle; LR, lateral rectus muscle; MR, medial rectus muscle; SO, superior oblique muscle; SR, superior rectus muscle; 1 , FTOZ craniotomy: cut through the orbital ridge lateral to the supraorbital foramen; 2 , trochlea within the trochlear fossa (ligamentous hypomochlion for the superior oblique muscle); 3, supraorbital foramen and supraorbital nerve; 4 , inferior orbital fissure; 5, frontal nerve (ophthalmic branch of the trigeminal nerve); 6, infraorbital nerve (maxillary branch of the trigeminal nerve); 7, FTOZ craniotomy MacCarty burr hole; 8, FTOZ craniotomy cut across the body of the zygoma, starting at the lateral orbital edge to the anterolateral edge of the inferior orbital fissure; 9 , lacrimal groove (accommodates the lacrimal sac and the nasolacrimal duct); 10, infraorbital foramen; 11 , anterior lacrimal crest. 


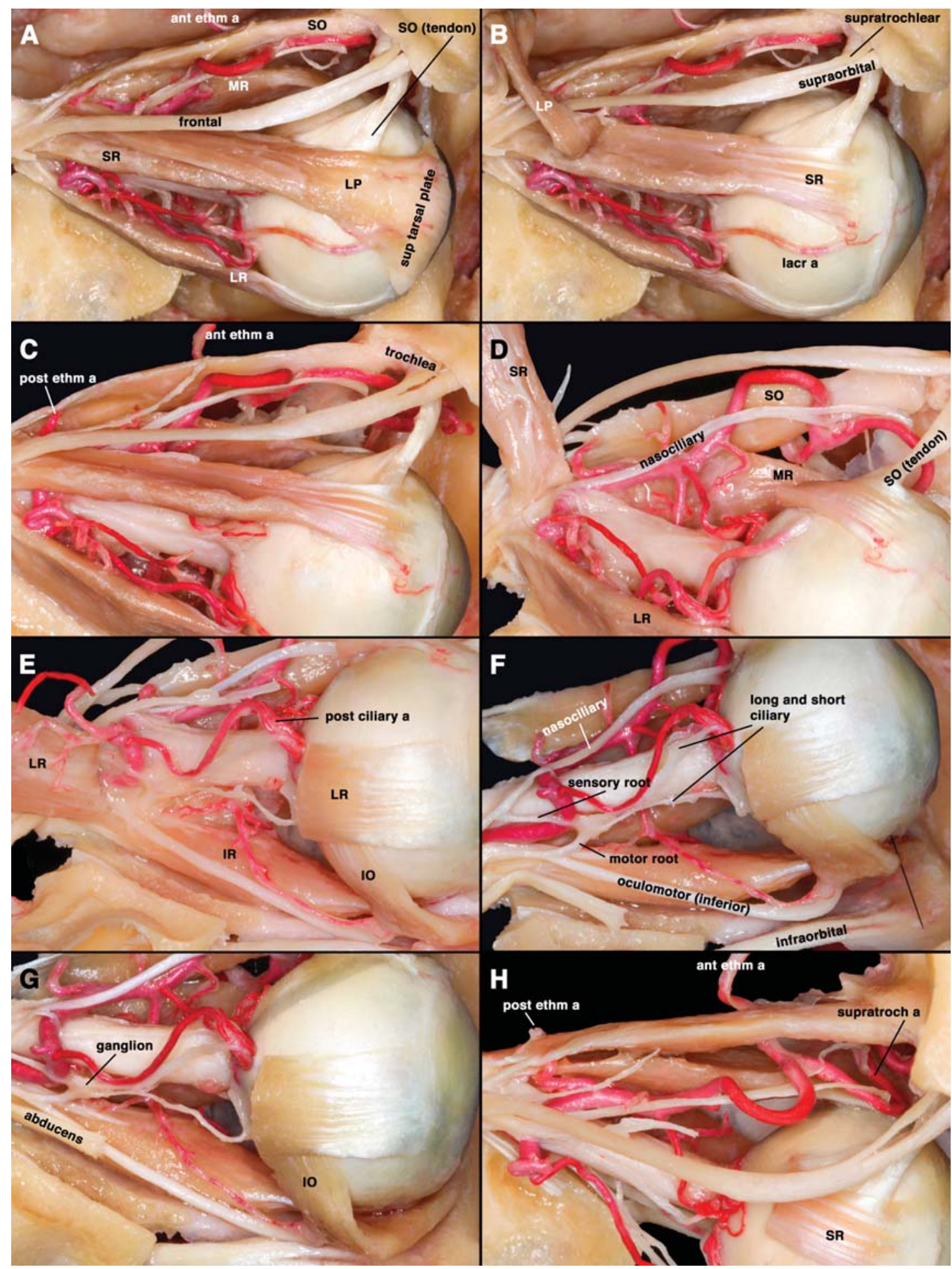

Fig. 9 Intraconal structures and extraocular muscles. In this figure, periorbita, intraconal fat, and periorbital structures have largely been removed for visualization. The right side of a formalin-fixed, silicone injected cadaveric specimen is shown from its lateral and superolateral aspects. (A) Levator palpebrae muscle and its aponeurosis, the superior tarsal plate. (B) Levator palpebrae muscle reflected to expose the superior rectus muscle. (C) Detail of the trochlea, the hypomochlion of the superior oblique muscle. (D) Superior rectus muscle reflected. (E) Lateral rectus muscle reflected to expose the ciliary ganglion. (F, G) Detail of the ciliary ganglion, its afferent and efferent branches, and the retrobulbar microvasculature. $(\mathrm{H})$ Course of the ophthalmic artery and its distal branches. ant ethma, anterior ethmoidal artery; ganglion, ciliary ganglion; IO, inferior oblique muscle; IR, inferior rectus muscle; lacr a, lacrimal artery; long and short ciliary, long and short ciliary nerves (sympathetic and parasympathetic postganglionic fibers); LP, levator palpebrae muscle; LR, lateral rectus muscle; motor root, motor root branching off the inferior division of the oculomotor nerve; MR, medial rectus muscle; post ciliary a, posterior ciliary arteries; post ethm a, posterior ethmoidal artery; SO, superior oblique muscle; SR, superior rectus muscle; sup tarsal plate, superior tarsal plate; sensory root, sensory root branching off the nasociliary nerve; supratroch a, supratrochlear artery; trochlea, trochlea within the trochlear fossa. 
The ophthalmic artery arises variably from the supraclinoidal ICA: $54 \%$ of the time from the anteromedial aspect, $38 \%$ from the superomedial aspect, and $7 \%$ from the medial aspect (rarely from the clinoidal or cavernous ICA segment). ${ }^{1}$ Rare, yet important, variations such as a fetal ophthalmic artery entering the orbit via the superior orbital fissure have been described (-Figs. 3, 5, and 7).

Between its origin and its entrance into the optic canal, the length of the ophthalmic artery averages 3 to $7 \mathrm{~mm}$. ${ }^{1}$ It enters the optic canal and courses within the optic nerve's dural sheath, inferomedial to the optic nerve in $41 \%$ of cases, directly inferior in $33 \%$, and inferolateral in $26 \%$ of cases. ${ }^{8} \mathrm{~A}$ recurrent branch might emerge from the ophthalmic artery to supply the intracanalicular, preforaminal, and cisternal segments of the optic nerve. Most often, the intracranial optic nerves are supplied by branches of the superior hypophyseal arteries.

The ophthalmic artery gives rise to the central retinal, long and short ciliary, supraorbital, medial and lateral palpebral, lacrimal, recurrent meningeal, ethmoidal, supratrochlear, and dorsal nasal arteries. The ophthalmic artery exits the optic canal and the annular tendon at their most lateral aspect, inferior to the intraorbital optic nerve. ${ }^{1}$ Among its proximal branches are the central retinal (see paragraph later) and lacrimal arteries, as well as some long ciliary arteries and variable muscular branches. The lacrimal artery courses anteriorly along the superior edge of the lateral rectus muscle. It gives rise to muscular branches, supply to the lacrimal gland and conjunctiva, and eventually to the lateral palpebral arteries. The ophthalmic artery steeply loops upward in the immediate proximity of the ciliary ganglion to pass over the optic nerve (in $85 \%$ of cases, and in $15 \%$, it crosses below the optic nerve) and to follow a more medial trajectory. ${ }^{2}$ It gives rise to the long and short ciliary arteries, some innominate muscular branches, and most notably the supraorbital artery, which courses anteriorly to exit the orbit via the supraorbital notch or foramen.

The ophthalmic artery continues anteromedially and gives off the posterior and anterior ethmoidal arteries which course inferior to the superior oblique muscle to exit the orbit via their respective ethmoidal foramina. From the endonasal perspective, the posterior ethmoidal artery courses in a medioposterior direction, whereas the anterior ethmoidal artery, which typically has a greater caliber, courses in a more medioanterior direction (-Figs. 3 and 9).

The distal ophthalmic artery then becomes the supratrochlear artery which parallels the course of the superior oblique and medial rectus muscles. The supratrochlear artery eventually exits the orbit below the trochlea, giving rise to the medial palpebral arteries, the dorsal nasal artery, and other smaller branches. ${ }^{2,6}$

The central retinal artery is the first branch of the ophthalmic artery and arises in proximity to the ciliary ganglion before the ophthalmic artery ascends steeply to cross over the optic nerve (-Figs. 5, 7 and 9). It pierces through the optic nerve's dural sheath to course anterior adjacent to and more distally within the optic nerve. The ophthalmic artery then gives rise to the short and long posterior ciliary arteries; these arteries enter the sclera in proximity to the optic nerve together with the long and short ciliary nerves. The anterior ciliary arteries (not shown) emerge from small arterial muscular branches and enter the sclera in the insertion zone of their respective extraocular muscles.

The ophthalmic artery also supplies the dura mater via lateral and medial systems and may thus partake in the supply of dural-based lesions. The lateral system includes branches of the lacrimal artery and the recurrent meningeal artery, an inconsistent branch that might arise from it to pass backward through the superior orbital fissure. The medial system consists of the anterior and posterior ethmoidal arteries that reach the anterior fossa through the ethmoidal canals.

\section{Venous Vasculature Associated with the Orbit}

The superior ophthalmic vein originates in the superomedial aspect of the anterior orbit then traverses the intraconal space between the optic nerve and the superior rectus muscle. It then continues its posterior cavernous-sinusbound course between the superior and the lateral rectus muscles to exit the orbit via the superolateral compartment of the superior orbital fissure.

The far more variable inferior ophthalmic vein drains the anteroinferior and lateral aspects of the orbit. It courses in the inferomedial quadrant, usually between the inferior and lateral rectus muscles, to exit the orbit underneath the annular tendon via the inferomedial compartment of the superior orbital fissure. It may eventually merge with the superior ophthalmic vein as it passes through the superior orbital fissure to drain into the cavernous sinus, and/or it may communicate with the pterygoid plexus via the inferior orbital fissure (-Figs. 5 and 6).

Both veins lie mainly within the muscular cone but exit the orbit outside the annular tendon. They also receive blood from the periorbital region, for example, the angular, supraorbital, supratrochlear, and facial veins.

The infraorbital vein, which chaperones the infraorbital nerve and artery on their course through the inferior orbital fissure, infraorbital canal, and infraorbital foramen, drains into the pterygoid plexus. The infraorbital vein, nerve, and artery all course in the orbital floor below the periorbita, and hence are not intraorbital structures in a strict sense. ${ }^{2,6}$

Conflict of Interest

None declared.

\section{References}

1 Lang J. Skull Base and Related Structures, 2nd ed. New York: Schattauer; 2001

2 Rhoton A. Cranial Anatomy and Surgical Approaches. Philadelphia, PA: Lippincott; 2003

3 Felding UA, Karnov K, Clemmensen A, et al. An applied anatomical study of the ethmoidal arteries: computed tomographic and direct measurements in human cadavers. J Craniofac Surg 2018;29(01):212-216 
4 Leblanc A. The Cranial Nerves, 2nd ed. Berlin: Springer; 1995

5 Lasjaunias P, Berenstein A, terBrugge KG. Surgical Neuroangiography, 2nd ed. Vol. 1. New York: Springer; 2001

6 Dutton J. Atlas of Oculoplastic and Orbital Surgery, 2nd ed. Philadelphia, PA: Lippincott; 2019
7 De Battista JC, Zimmer LA, Theodosopoulos PV, Froelich SC, Keller JT. Anatomy of the inferior orbital fissure: implications for endoscopic cranial base surgery. J Neurol Surg B Skull Base 2012;73 (02):132-138

8 Renn WH, Rhoton AL Jr. Microsurgical anatomy of the sellar region. J Neurosurg 1975;43(03):288-298 